\title{
Predicted Ice Shape Formations on a Boundary Layer Ingesting Engine Inlet
}

\author{
Christopher Porter NASA Glenn Research Center
}

Citation: Porter, C., “Predicted Ice Shape Formations on a Boundary Layer Ingesting Engine Inlet," SAE Technical Paper 2019-01-2025, 2019.

\section{Abstract}

omputational ice shapes were generated on the boundary layer ingesting engine nacelle of the D8 Double Bubble aircraft. The computations were generated using LEWICE3D, a well-known CFD icing post processor. A 50-bin global drop diameter discretization was used to capture the collection efficiency due to the direct impingement of water onto the engine nacelle. These discrete results were superposed in a weighted fashion to generate six drop size distributions that span the Appendix $\mathrm{C}$ and $\mathrm{O}$ regimes. Due to the presence of upstream geometries, i.e. the fuselage nose, the trajectories of the water drops are highly complex. Since the ice shapes are significantly correlated with the collection efficiency, the upstream fuselage nose has a significant impact on the ice accretion on the engine nacelle. These complex trajectories are caused by the ballistic nature of the particles and are thus exacerbated as particle size increases. Shadowzones are generated on the engine nacelle, and due to the curvature of the nose of the aircraft the shadowzone boundary moves from lower inboard to upper outboard as particle size increases. The largest particle impinging one the engine nacelle from the 50-bin discretization was the $47 \mu \mathrm{m}$ drop diameter. As a result, the MVD greater than $40 \mu \mathrm{m}$ Appendix $\mathrm{O}$ conditions were characterized by extremely low collection efficiency on the engine nacelle for these direct impingement simulations.

\section{Introduction}

$$
\text { . }
$$

he Advanced Air Transport Technology project of the Advanced Air Vehicles Program under the Aeronautics Research Mission Directorate at NASA has an ongoing interest in developing revolutionary next generation $(\mathrm{N}+3)$ commercial transport designs that are energy efficient and environmentally compatible [1]. To achieve these goals the $\mathrm{N}+3$ aircraft employ designs that are atypical of current commercial transport vehicles. One of these atypical design features is boundary layer ingesting (BLI) engines. BLI engines can be seen in three of the $\mathrm{N}+3$ geometries depicted in Fig. 1 , specifically the D8 "Double Bubble, the STARC-ABL (tail "pusher" fan), and the NX3.

There are three primary ways to investigate the icing risk of airframe geometries: 1) flight testing, 2) icing tunnel testing, and 3) computational simulation. Flight testing theoretically offers the best results, as the full scale aircraft can be flown through realistic conditions. However, this requires a full scale aircraft to be designed and built as well as seeking transient icing weather conditions as they occur in nature. Due to the cost associated with these steps, this method is not necessarily desirable for the evaluation of aircraft that are in the early design stages of development.

Icing tunnel testing attempts to simulate flight testing. It has advantages over flight testing, such as providing the user control over the operating condition and repeatability. However, tunnel testing restricts model size to some fraction
FIGURE 1 Five Various N+3 Geometries. Top (Left to Right): D8 “Double Bubble”, HWB, SURGAR VOLT - Bottom (Left to Right): STARC-ABL, N3X

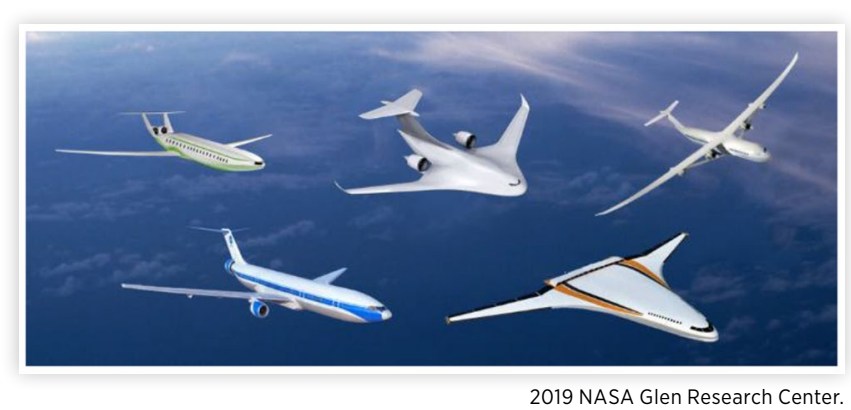

of the test section size of the tunnel in which it is being tested. Due to scaling complexities associated with the icing phenomenon, generating a representative scaled geometry and test matrix is both time consuming and costly.

Computational icing tools attempt to replicate both of the above. However, the icing problem is an unsteady, turbulent, multi-scale, and multi-phase phenomenon. Simulating all of the physical effects that contribute to airframe icing with currently available computational resources is not feasible. Therefore, these computational icing tools only attempt to capture the first order, or dominant, effects so that simulations can be computed in time frames conducive for research and development, while still providing a reasonable solution. Because these tools only approximate the water impingement 
of a droplet distribution, as well as the resulting ice shape, the tools must be sufficiently validated to be used in a predictive fashion.

LEWICE3D [2] is a quasi-3D ice accretion solver, and for straight wings, is nearly indistinguishable from LEWICE, a two dimensional ice accretion solver that is considered a validated production level code []ㅡ with respect to the Title 14 CFR Part 25 Appendix C [4] regime. Due to the degrees of freedom a third dimension adds to the problem, as well as the Supercooled Large Drops found in Title 14 CFR Part 25 Appendix $O$ [5], there are aspects of LEWICE3D that aren't sufficiently validated. However, there is still usefulness in LEWICE3D analyses even outside the validated predictive range as it provides cost-effective insight into a complex problem. For instance, LEWICE3D has been used in the past to assess wing-only $[\underline{6}, \underline{7}]$, engine inlet $[\underline{8}]$, as well as classical wing body configurations $[\underline{7}, \underline{9}, \underline{10}]$.

One of the computational modeling complexities that can arise is the splashing problem. It has been shown that the splashing of large drops can contribute to reduced collection efficiency in the regions aft of the stagnation point [7]. The same study illustrated that the splashing model in LEWICE3D v. 3 provided collection efficiency results that agreed more favorably with experiment than LEWICE3D results without the splashing model.

Another computational complexity that can arise is the influence upstream geometries have on downstream collection efficiencies. A simulation of the NASA S3 aircraft [10] suggests that the upstream geometry "hides" the downstream surfaces from water impingement, generating what the author called a shadowzone. At the edges of these shadowzones are regions of high collection efficiency where "a concentrating effect or compression effect on the particles, similar to streamlines traversing the leading edge region of a wing" occur. It was noted that this phenomenon primarily affects the larger drop diameters in the distributions.

This paper discusses the details of the D8 geometry, the computational tools used, and the aerodynamic operating conditions simulated in an effort to predict the ice that would form on the BLI engine nacelle. The icing conditions simulated span a variety of freestream static temperatures with liquid water contents pertaining to the Continuous-Maximum (17.4 nautical miles) condition as described by the FAA in Title 14 CFR Part 25 Appendixes C [4] and O []‥ The predicted ice shapes are compared purely on their geometrical size, with the idea being that larger ice masses pose a higher shedding threat to the downstream fan blades.

\section{Geometry and Simulation Details}

\section{Geometry}

The geometry being assessed in this paper is the D8 "Double Bubble" aircraft [11]. Its design is the result of a NASA sponsored joint effort between Aurora Flight Sciences, MIT and Pratt \& Whitney. There are multiple variants of the D8 design.
FIGURE 2 D8 “Double Bubble” Computational Surface Geometry.

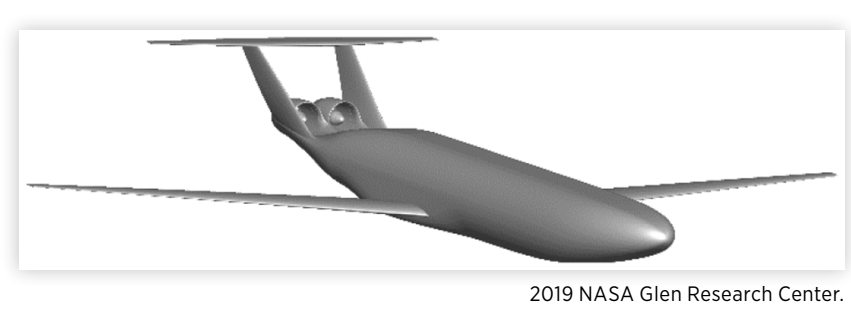

The design provided for this effort, pictured in Figs. 1 and $\underline{2}$, consisted of a two engine configuration rather than the three engine configuration seen in previous mock-ups. This design has a half-span of $75 \mathrm{ft}$. (900 in.), a body length of $114.9 \mathrm{ft}$. (1379 in.) and a mean chord length of $10 \mathrm{ft}$. (120 in.).

\section{Simulation}

Operating Conditions, Mesh, and CFD Solver Details The flight operating condition simulated is one that is representative of a typical hold pattern for commercial aircraft. The hold pattern is the portion of the flight where the aircraft is exposed to icing conditions for extended periods of time. It should be noted that this representative hold condition is almost assuredly not the exact hold condition for this particular aircraft. Determining that specific hold condition would have required additional aerodynamic analysis. The purpose of this study is to assess the icing risks for the D8 aircraft. Thus, utilization of a representative operating condition, and not necessarily the exact operating condition, was deemed sufficient to meet that goal. Details of the simulated flight condition are listed in Table 1.

The mesh contained 423 overset blocks, with each block consisting of a structured mesh with hexahedral cells. The number of grid points in the entire mesh was on the order of 288 million. The domain boundaries were placed well in the freestream, roughly 50 body lengths away from the aircraft. A first cell height of $y+\approx 1$ was targeted, and achieved on most of the body surfaces. Y+ is a non-dimensional quantity that describes the boundary layer, with values of 1 considered to be resolved. X-plane and Y-plane cuts of the near body overset mesh are shown in Fig. 3.

The flow simulations were completed by H. Doğuş Akaydin and Shishir Pandya at NASA Ames Research Center. The CFD solver utilized was the in-house NASA solver, Overflow, version 2.2k [12]. It is a Reynolds Averaged Navier Stokes solver with a variety of flux and turbulence schemes.

TABLE 1 Simulated Operating Condition for the D8 "Double Bubble".

\begin{tabular}{l|l}
\hline$V_{\infty}$ (mph) & 200 \\
\hline Altitude (ft.) & 10,000 \\
\hline AoA (deg.) & 4 \\
$M_{\infty}$ & 0.272 \\
$\mathrm{Re}_{\infty}$ & $14,511,360$
\end{tabular}

2019 NASA Glen Research Center. 2019 NASA Glen Research Center. 
FIGURE 3 X-plane and Y-plane Cuts of the Near Body Overset Mesh.

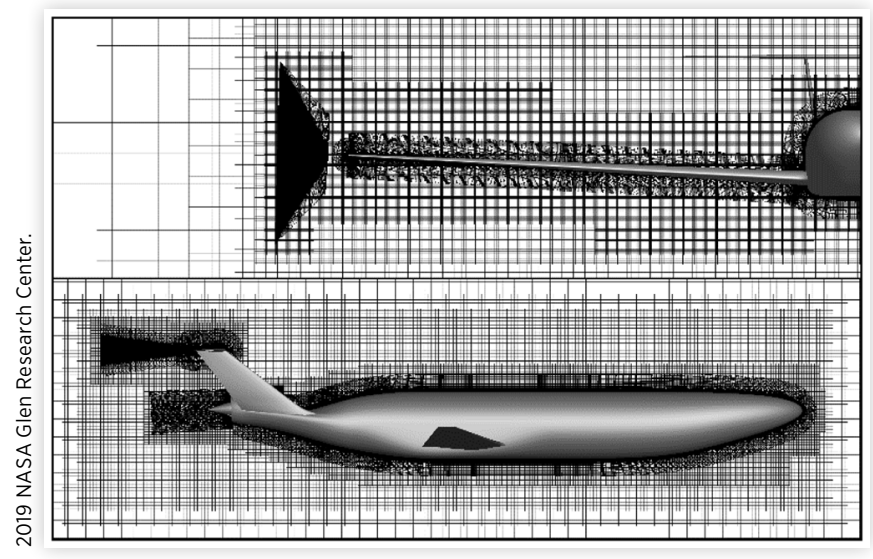

FIGURE 4 Lift Coefficient and Drag Coefficient Convergence Histories.

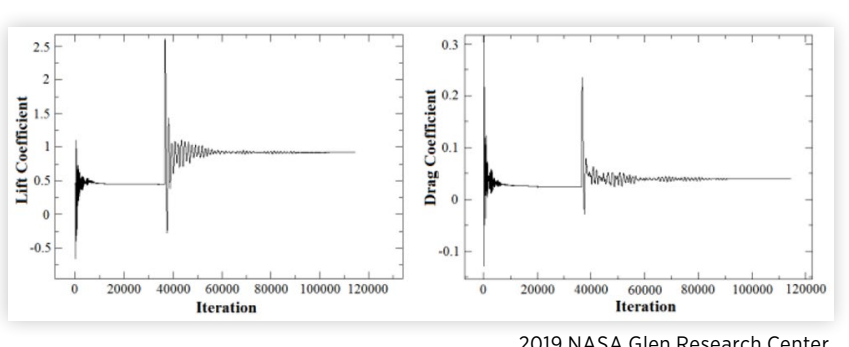

2019 NASA Glen Research Center.

A $2^{\text {nd }}$ order central finite difference scheme was employed, along with the SST turbulence model. The SST turbulence model is a blended model that utilizes a k- $\omega$ scheme in the near wall region, a $\mathrm{k}-\varepsilon$ scheme in the free shear region, and a blending function to smoothly transition between the two [13]. The lift coefficient and drag coefficient histories are shown in Fig. 4, and illustrate that some level of convergence was achieved on the finest multi-grid level.

The icing operating conditions were chosen to be representative of the Continuous-Maximum (17.4 nautical miles) condition as described by the FAA in Title 14 CFR Part 25 Appendix C [ 4 ] and $\mathrm{O}[\underline{5}]$ for a variety of thermal conditions. The data was extracted from figures of the icing envelopes using a plot digitizer and is tabulated in Table 2. The icing

TABLE 2 Liquid Water Content for the Various Drop Diameter Distributions for a Variety of Static Temperatures for Continuous-Maximum Icing Events.

\begin{tabular}{|l|l|l|l|l|}
\hline & $\mathbf{0}{ }^{\circ} \mathbf{C}$ & $-\mathbf{- 1 0}{ }^{\circ} \mathbf{C}$ & $-\mathbf{2 0}{ }^{\circ} \mathbf{C}$ & $\mathbf{- 3 0}{ }^{\circ} \mathbf{C}$ \\
\hline Langmuir D (20 um) & 0.637 & 0.425 & 0.211 & 0.140 \\
\hline Langmuir D (40 um) & 0.150 & 0.100 & 0.060 & 0.040 \\
\hline FZDZ < 40 um & 0.439 & 0.374 & 0.317 & n/a \\
\hline FZDZ > 40 um & 0.270 & 0.230 & 0.195 & n/a \\
\hline FZRA < 40 um & 0.259 & 0.221 & n/a & n/a \\
\hline FZRA > 40 um & 0.310 & 0.264 & n/a & n/a \\
\hline
\end{tabular}

events in this paper were simulated by a 45 minute exposure to capture the "worst case" ice shapes for these icing conditions.

LEWICE3D Details LEWICE3D version 3.63 was used for this analysis. LEWICE3D is a CFD post processor that utilizes the computed steady-state q-vector to calculate Lagrangian particle trajectories through the flow field. It should be noted, however, that this Lagrangian method isn't tracking individual drops. Instead, each computed trajectory represents a stream tube of a small finite area. The collection efficiency is then computed as the freestream area of the impacting stream tubes divided by the area of the surface cell. This method requires a sufficiently resolved stream tube discretization to ensure numerical accuracy. In order to achieve the required refinement, LEWICE3D employs a quadtree approach to self-resolve the trajectory field. By framing the problem in this fashion, computational time is greatly reduced.

In order to compute continuous droplet distributions, the distribution is discretized into bins with each bin having a representative discrete drop diameter, typically the MVD of that bin. Each bin is simulated independently by computing the impingement characteristics of the representative droplet size. The resulting collection efficiencies of all of the bins are superposed in a weighted fashion. The weighting function is equal to the fractional amount of the TWC each bin contributes to the distribution.

It should be noted that these simulations were run absent of the splashing model. While the splashing model has the potential to be more accurate, comparisons in Ref.[7] only validated the loss of mass on wing sections due to splashing. The inclusion of the splashing model would almost assuredly change the collection efficiency results on the engine inlet due to the inclusion of re-entrained splashed water from the nose of the aircraft. However, the splashing model has never been validated for the re-entrainment of liquid water and the subsequent impingement of the re-entrained water on downstream geometries. Thus, the results presented here can be thought of as the icing that occurs due to direct impingement.

LEWICE3D also computes the mass and energy balance of the surface water that enables the prediction of ice shapes for various thermal operating conditions. An assumption is made that small changes in temperature have negligible effect on the velocity vector field. Thus only one trajectory computation is performed for all thermal conditions. The icing algorithms of LEWICE3D are quasi-3d algorithms that use a strip theory approach. The user specifies one (or more) two dimensional planes in three space that intersect the geometry. The mass and energy balance, liquid water runback, and resulting ice growth occur within these two dimensional planes using the Messinger model [14]. A process exists to loft the results from multiple strips into a three dimensional ice growth. However, that process is not utilized in this paper.

Icing Strip Cut Planes For each thermal condition, multiple strip-based ice accretions were simulated. The specific discretization of the cut planes was chosen such that it spanned a majority of the collection efficiency and captured the theta variation of the results while limiting the overall amount 
FIGURE 5 Ice Accretion Cut Planes on the Engine Inlet of the D8 "Double Bubble" Aircraft.

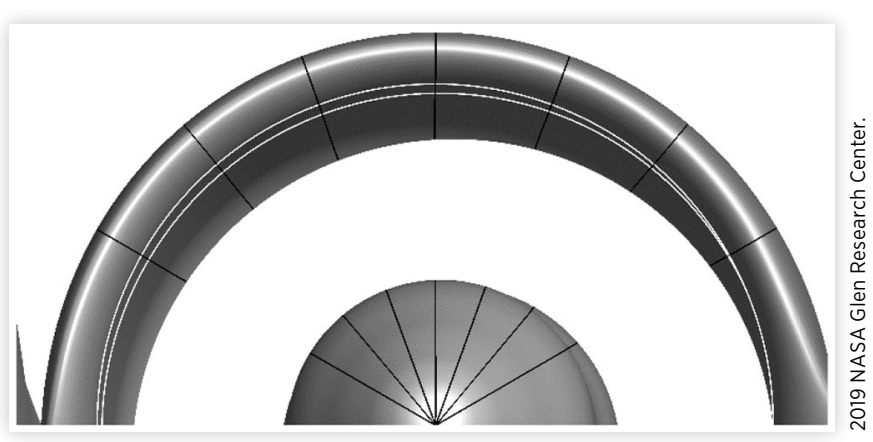

of cuts. The result being seven cut planes, all of which pass through the spinner centerline axis of $[y, z]=[42.0 ”, 40.25 ”]$. These cut planes occur in 20 degree increments ranging from 30 degrees above the horizontal to 150 degrees above the horizontal and are illustrated in Fig. 5.

Droplet Distributions Six distributions were chosen for this study as described in Table 2. The first two distributions are 20 micron and 40 micron MVD distributions. These distributions are representative of the Title 14 CFR Part 25 Appendix C [4] Continuous Maximum (Stratiform Clouds) and Intermittent Maximum (Cumuliform Clouds) icing conditions, respectively. The distributions themselves are Langmuir " $D$ " [15], which is a commonly used distribution for Appendix $\mathrm{C}$ conditions [16]. It should be noted that the Langmuir " $D$ " distribution is a discretized distribution and numerically documented by its 7-bin representation. However, a higher resolution of the Langmuir " $D$ " curve is desired for the purposes of this paper. Reference [17] depicts a figure of the discretized Langmuir " $D$ " distribution along with a continuous representation of the distribution. It is not clear where the continuous distribution originated or how it is calculated. However, a cubic spline representation of the 7-bin Langmuir " $\mathrm{D}$ " distribution, along with the implied boundary condition at $\mathrm{d}_{\mathrm{i}} / \mathrm{MVD}=0$ and an assumed boundary condition at $\mathrm{d}_{\mathrm{i}} /$ $M V D=3$, produced a continuous curve that was visually identical to that depicted graphically in Ref. [17]. This high resolution, cubic spline representation of the Langmuir " $D$ " distribution was used to generate the higher sampled discrete distributions seen in this paper. The nine data points used for the cubic spline are listed in Table 3 . Note that the cumulative mass tabulated here is an increasing function rather than the decreasing function depicted in Ref. [17]. This was done to maintain consistency with Title 14 CFR Part 25 Appendix O [ㄷ] where the distributions are depicted as increasing functions.

The other four distributions are graphically documented in Appendix O [5]. They are the "Freezing Drizzle MVD Less than $40 \mu \mathrm{m}$ ", "Freezing Drizzle MVD Greater than $40 \mu \mathrm{m}$ ", "Freezing Rain MVD Less than $40 \mu \mathrm{m}$ ", and the "Freezing Rain MVD Greater than $40 \mu \mathrm{m}$ " distributions. Highly resolved (one micron increment) numerical representations of these distributions were obtained from the FAA William J. Hughes Technical Center.

TABLE 3 The Nine Data Points Used to Generate the Cubic Spline Continuous Langmuir "D” Curve.

\begin{tabular}{|c|c|}
\hline $\mathbf{d}_{\mathrm{f}} / \mathrm{MVD}$ & Cumulative Mass \\
\hline 0.000 & 0.000 \\
\hline 0.310 & 0.025 \\
\hline 0.520 & 0.100 \\
\hline 0.710 & 0.250 \\
\hline 1.000 & 0.500 \\
\hline 1.370 & 0.750 \\
\hline 1.740 & 0.900 \\
\hline 2.220 & 0.975 \\
\hline 3.000 & 1.000 \\
\hline
\end{tabular}

Droplet Distribution Discretization The distributions being simulated in this document consist of drop diameter ranges that span three orders of magnitude. The array of drop diameters chosen to discretize these ranges is a 50-bin integer approximation of a $12.5 \%$ growth curve with an initial droplet diameter of $5 \mu \mathrm{m}$. In other words, the $i+1$ bin has a drop diameter that is $12.5 \%$ larger than the $\mathrm{i}^{\text {th }}$ bin, with the initial $(i=1)$ bin being set to a drop diameter of $5 \mu \mathrm{m}$. This discretization method was chosen to ensure a smooth increase in momentum from bin to bin. The formula for this distribution can be seen in Eq. 1 where $d$ represents the droplet diameter in microns and $i$ represents the bin index. For clarity, please note that the [\rceil symbols represent the ceiling function, which maps the argument to the least integer greater than or equal to the argument, i.e. it rounds the argument up.

$$
d_{i}=\left\lceil 5(1.125)^{(i-1)}\right\rceil
$$

Portions of both the exact and rounded growth distributions can be seen in Table 4 with the resulting drop diameters illustrated graphically in Fig. 6. Note the slight "hump" in the data at lower drop diameters $(<20 \mu \mathrm{m})$ that is most visible on the logarithmic plot in Fig. 6. This is an artifact of the integer rounding being more significant in relative magnitude at the lower drop diameters. At the higher drop diameters, where this rounding is less significant in relative magnitude, the integer based discretization more closely approximates the exact $12.5 \%$ growth curve.

The graphs in the left column of Figs. 7 and $\underline{8}$ illustrate the projection of the 50-bin discretization shown in Fig. 6

TABLE 4 50-Bin Discrete Drop Diameter Distribution. Both the Exact $12.5 \%$ Growth Array ( $2^{\text {nd }}$ Column) and its Integer Approximation ( $3^{\text {rd }}$ Column) are Pictured.

\begin{tabular}{|c|c|c|}
\hline Bin Index & Droplet Diameter $(\mu \mathrm{m})$ & Droplet Diameter $(\mu \mathrm{m})$ \\
\hline 1 & 5 & 5 \\
\hline 2 & 5.625 & 6 \\
\hline 3 & 6.328125 & 7 \\
\hline$\vdots$ & $\vdots$ & $\vdots$ \\
\hline 48 & 1268.056368 & 1269 \\
\hline 49 & 1426.563414 & 1427 \\
\hline 50 & 1604.883841 & 1605 \\
\hline
\end{tabular}


FIGURE 6 50-Bin Discrete Drop Diameter Distribution. Left- Linear Y-Axis. Right: Logarithmic Y-Axis.

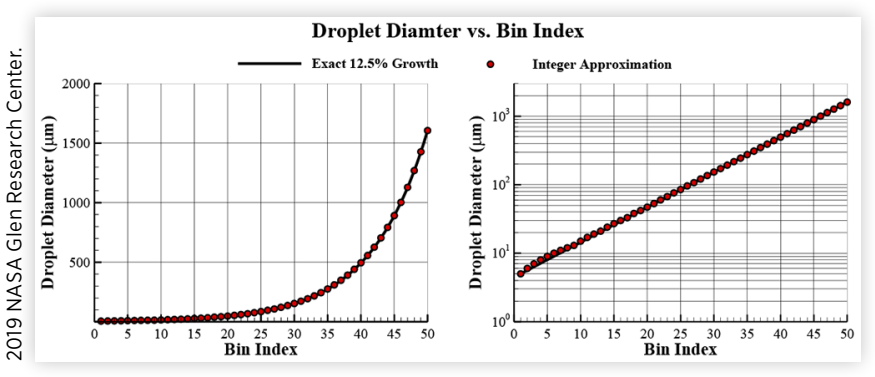

FIGURE 7 50-Bin Representation of Various Drop Diameter Distributions. Left: Discretization of the Continuous Distributions. - Rights: Amount of Total Water Content in Each Bin.
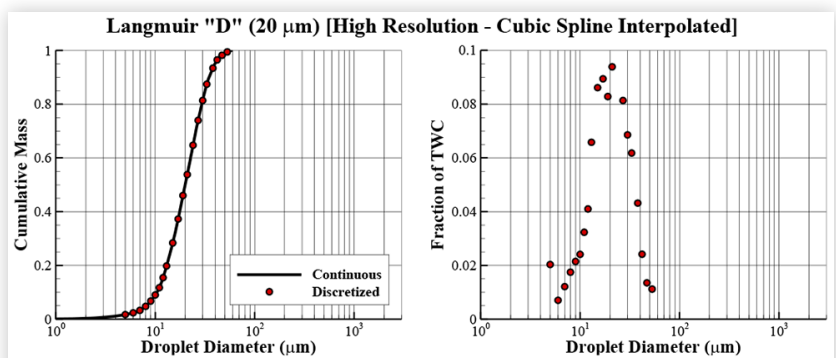

Langmuir "D" (40 $\mu \mathrm{m})$ [High Resolution - Cubic Spline Interpolated]
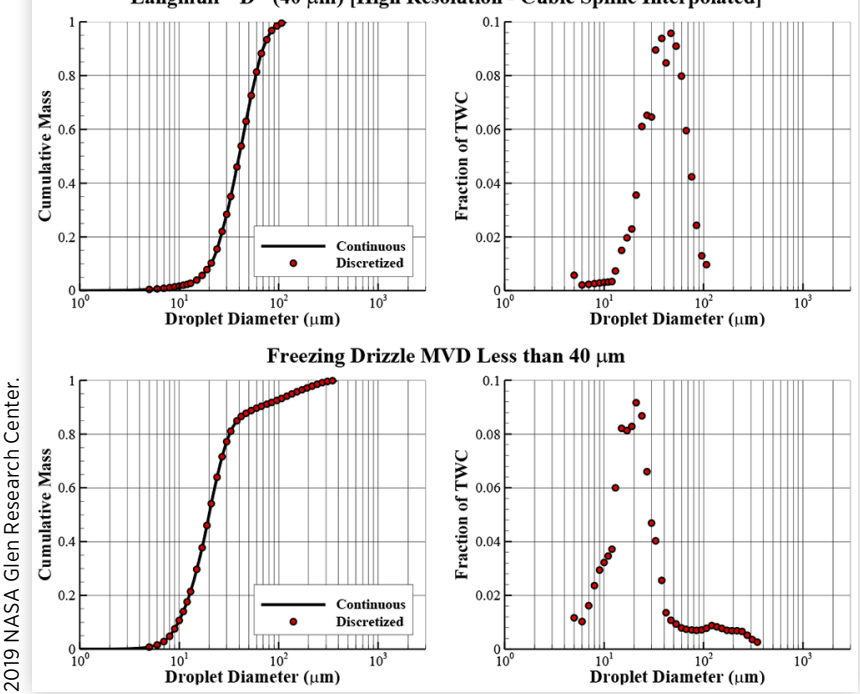

onto the six desired droplet distributions. The graphs in the right column of Figs. 7 and $\underline{8}$ illustrate the fractional TWC contained in each bin. These fractions are the weights used in the super position algorithm of LEWICE3D.

\section{Results}

Collection efficiency is the prerequisite data set required to generate ice shapes on an aerodynamic geometry. The collection efficiency for the six drop diameter distributions
FIGURE 8 50-Bin Representations of Various Drop Diameter Distributions. Left: Discretization of the Continuous Distributions. - Right: Amount of Total Water Content in Each Bin.
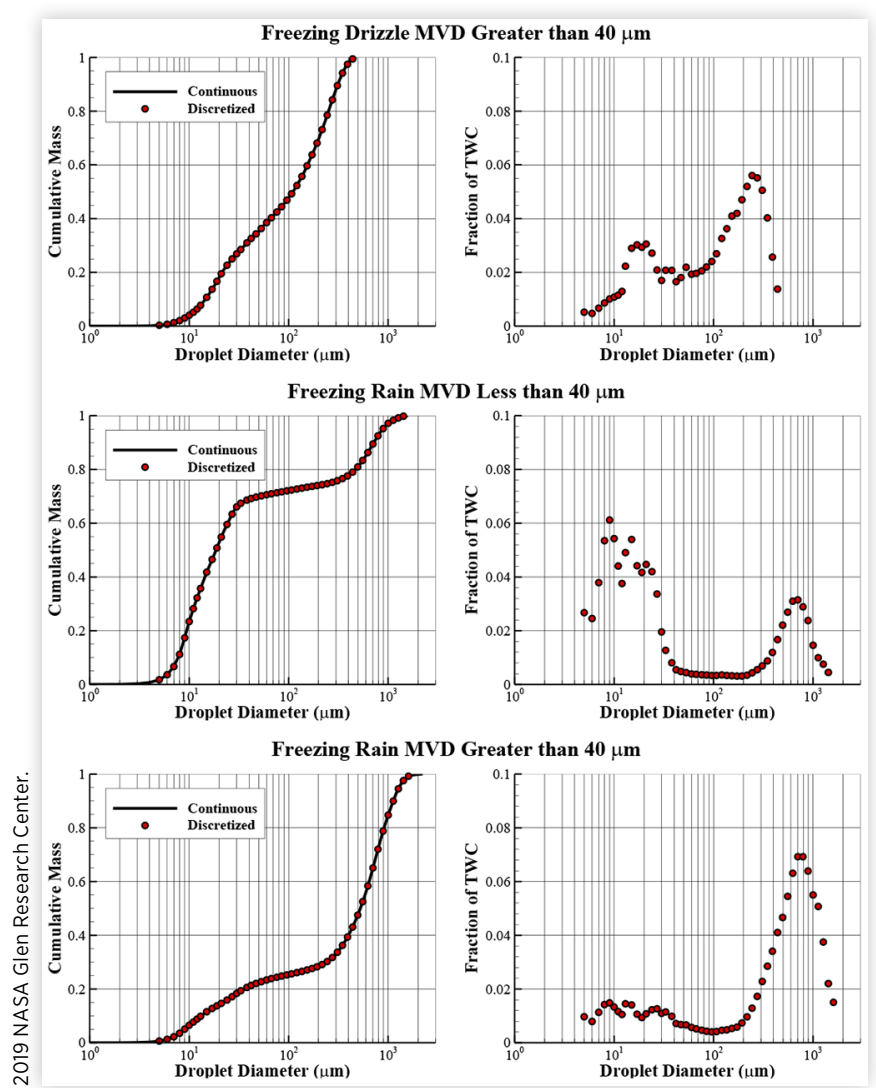

representative of Appendix $\mathrm{C}$ and $\mathrm{O}$ icing conditions can be seen in Fig. 9. Since the effect of temperature on the velocity field is assumed to be negligible, one collection efficiency result is used for all thermal conditions. Due to the $4^{\circ}$ angle of attack of the aircraft the boundary layer ingesting engines are hidden and only particles $47 \mu \mathrm{m}$ and under can navigate around the nose of the D8 and impinge on the inlet of the engine nacelle. The larger particles are more ballistic in nature and are unable to follow the flow around the nose and impinge on the engine inlet. As such the Appendix $\mathrm{O}$ conditions with significant water content at drop diameters above $47 \mu \mathrm{m}$ are characterized by very low collection efficiency on the engine inlet. Note that these simulations do not include splashing due to no validation data existing for this particular geometric configuration (e.g. re-entrainment of water due to splashing and the subsequent impingement of the re-entrained water on downstream geometries such as a boundary layer ingesting engine inlet). The inclusion of splashing would likely increase the collection efficiency on the engine inlet for these cases with significant water content at large drop diameters.

A more detailed discussion of the collection efficiency results for the D8 "Double Bubble" can be found in a prior paper by Porter and Potapczuk [18]. This paper not only discusses the drop diameter distributions seen here, but also discusses results of a select group of discrete drop diameters 
FIGURE 9 Collection Efficiencies on the Engine Inlet for a Variety of Cloud Distributions. (Top to Bottom: Langmuir "D” $20 \mu \mathrm{m}$ - Cubic Spline, Langmuir "D” $40 \mu \mathrm{m}$ - Cubic Spline, Freezing Drizzle MVD Less than $40 \mu \mathrm{m}$, Freezing Drizzle MVD Greater than $40 \mu \mathrm{m}$, Freezing Rain MVD Less than $40 \mu \mathrm{m}$, Freezing Rain MVD Greater than $40 \mu \mathrm{m}$ )

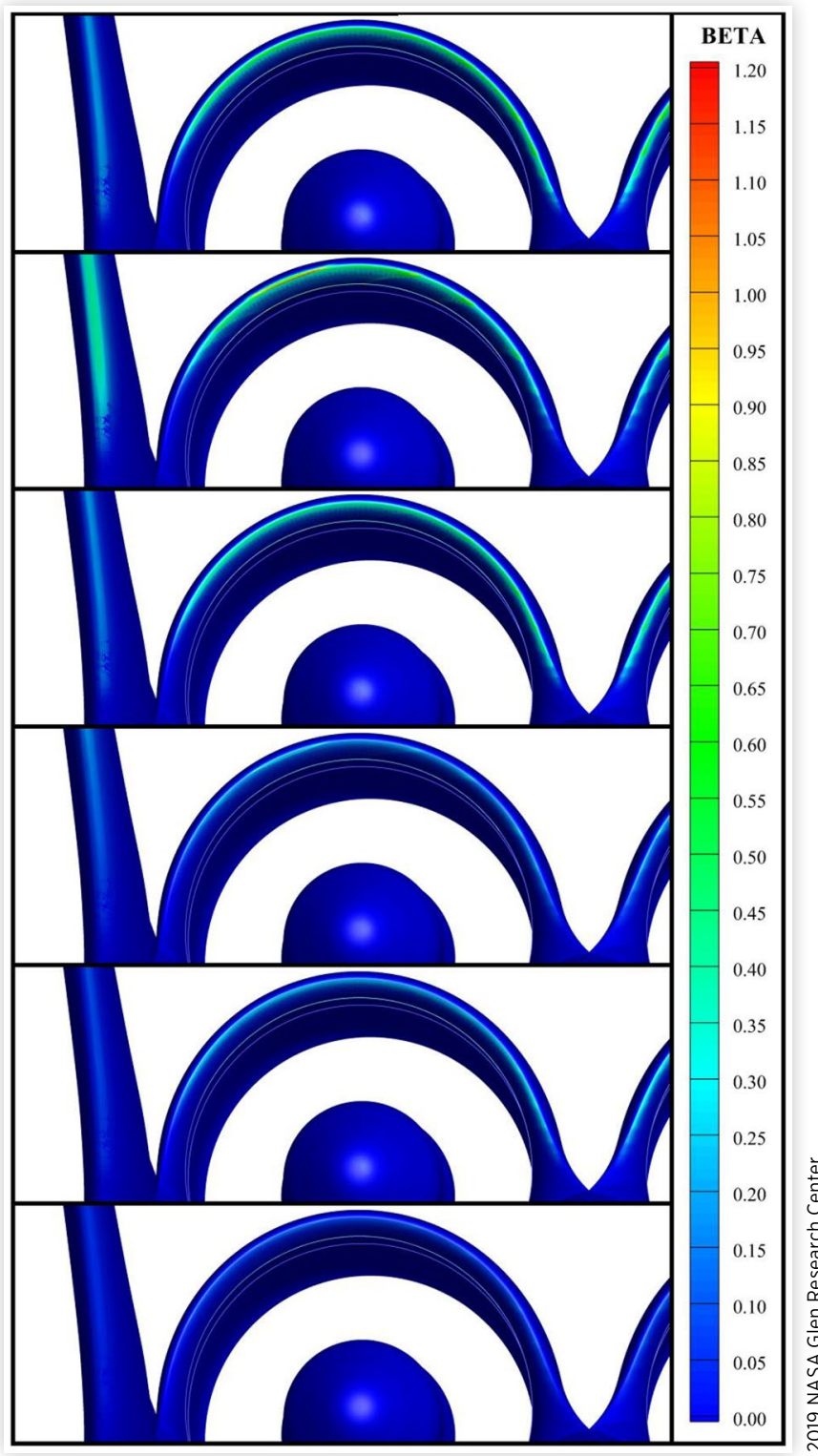

to understand how the water collection characteristics of the geometry change with drop diameter.

The ice shapes depicted in this paper will be presented as two dimensional figures viewed normal to the cut plane. For ease of readability, the data of each cut plane is rotated about the spinner centerline onto a common reference plane. The clean surface of the engine inlet for each cut plane is illustrated in Fig. 10. As can be seen, there is a slight variation of the engine nacelle in the region of the icing limits. The variation is subtle enough that the ice shapes from various circumferential cuts can be plotted on the same figure.

It should be noted that the lower surface of the inlet terminates at the computational engine inlet face, artificially
FIGURE 10 Clean Engine Inlet Profiles for the Various Circumferential Cut Planes. Units of the Unlabeled Axes are Inches.

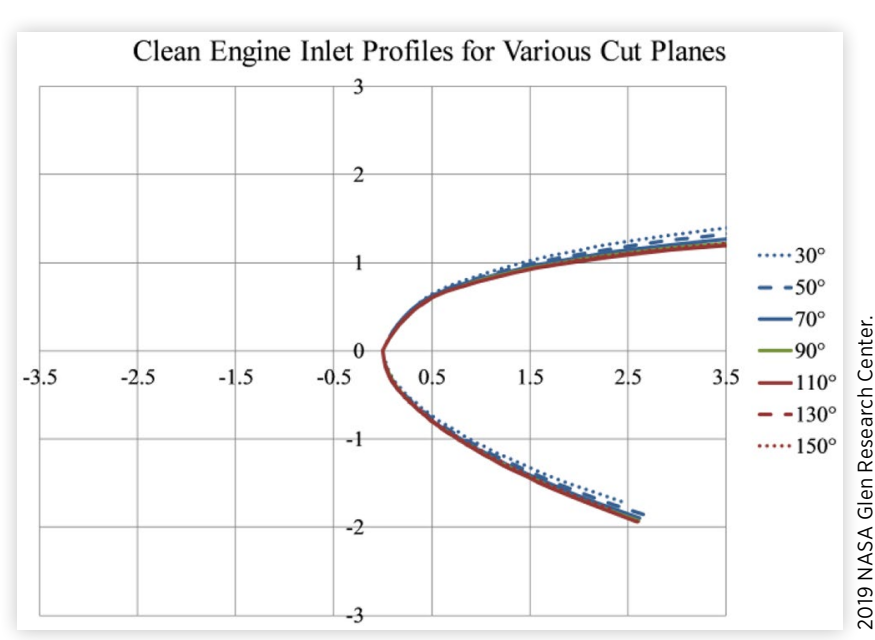

truncating the extent of the icing limits. This is because the engine is not modeled directly. Instead an asymmetric computational outflow boundary is placed at the inlet near where the fan face would be to force the correct asymmetric mass flow entering the engine and a computational inlet boundary is placed downstream to capture this mass being exhausted from the nozzle. Thus, the region directly aft of the engine inlet (the outflow boundary) is outside the computational domain. One might suggest to move this outflow plane further downstream to capture the actual icing limits, however, this region of the engine inlet would be influenced by the presence of the fan which is neglected computationally.

Figure 11 illustrates a temperature sweep on the ice shapes generated for the Langmuir " $D$ " $20 \mu \mathrm{m}$ distribution at the vertical cut plane. Note that while the $0^{\circ} \mathrm{C}$ condition contains the highest liquid water content, the ice shape generated is

\section{FIGURE 11 Simulated Ice Shapes of Various Thermal} Conditions for the Langmuir "D" $20 \mu \mathrm{m}$ Distribution on the $90^{\circ}$ Circumferential Cut Plane. Units of the Unlabeled Axes are Inches.

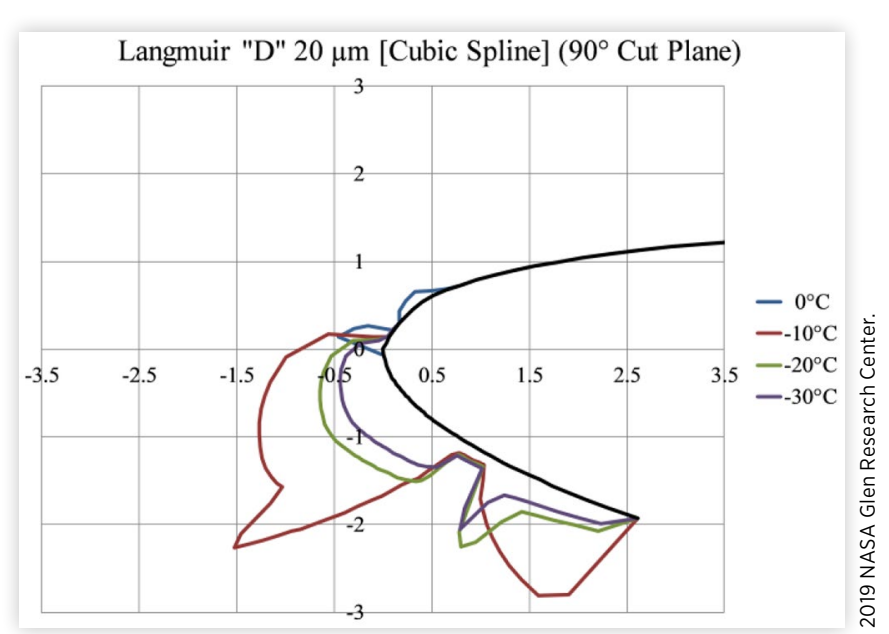


extremely small and likely benign due to the high thermal content, leading to an inability to freeze the impinging water. At $-10^{\circ} \mathrm{C}$, however, the impinging water (and any resulting runback) freezes. Since the liquid water content decreases as a function of temperature, as seen in Table 2 , the $-10^{\circ} \mathrm{C}$ thermal condition produces the largest ice shapes for all drop diameter distributions tested.

The reader should note that the ice shape is computationally truncated where the mass outflow boundary ends the computational domain. This is visually apparent for the backward facing step formed for the $-10^{\circ} \mathrm{C}$ thermal condition, but can also be seen more subtly for the two colder thermal conditions as well. As previously mentioned the icing physics in the region local to the fan would be greatly affected by the presence of the fan, and moving the computational boundary further downstream would not necessarily produce a more accurate icing result for the real world condition. However, the reader should note that the ice shape almost assuredly does not form a backward facing step in this region, and that this formation of the backward facing step is purely an artifact forcing ice growth to be zero at that final computational node since no other information exists that leads to a water tight ice shape.

Figure 12 illustrates a drop diameter distribution sweep at the $-10^{\circ} \mathrm{C}$ thermal condition on the vertical cut plane. Two of the distributions have significantly more ice mass than the other four distributions. These two distributions are the Langmuir "D" $20 \mu \mathrm{m}$ distribution and the Freezing Drizzle MVD Less than $40 \mu \mathrm{m}$. Examining Fig. 9, however, there are three distributions that are characterized by significant collection efficiency, the previously mentioned two distributions as well as the Langmuir " $\mathrm{D}$ " $40 \mu \mathrm{m}$ distribution. Table 2 provides the reason for the lack of ice mass for the Langmuir " $D$ " 40 $\mu \mathrm{m}$ case, a significantly lower liquid water content than all other distributions for the Continuous Maximum condition. Because of this lower liquid water content, the resulting ice mass for the Langmuir " $D$ " $40 \mu \mathrm{m}$ distribution is comparable

FIGURE 12 Simulated Ice Shapes of Various Drop Diameter Distributions for the $-10^{\circ} \mathrm{C}$ Thermal Operating Condition on the $90^{\circ}$ Circumferential Cut Plane. Units of the Unlabeled Axes are Inches.

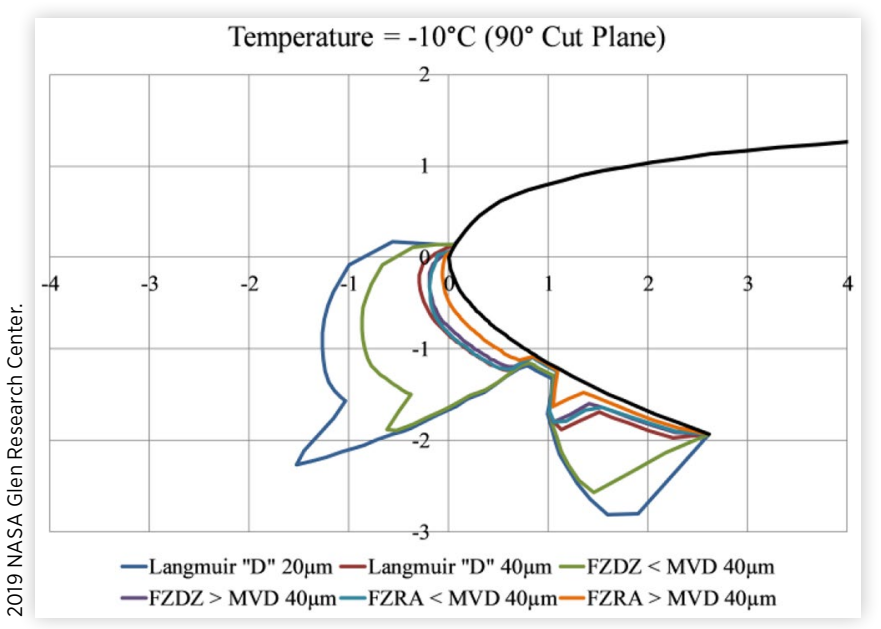

2019 NASA Glen Research Center
FIGURE 13 Simulated Ice Shapes of Various Cut Planes for the $-10^{\circ} \mathrm{C}$ Thermal Operating Condition and Langmuir " $\mathrm{D}$ " 20 $\mu \mathrm{m}$ Distribution. Units of the Unlabeled Axes are Inches.

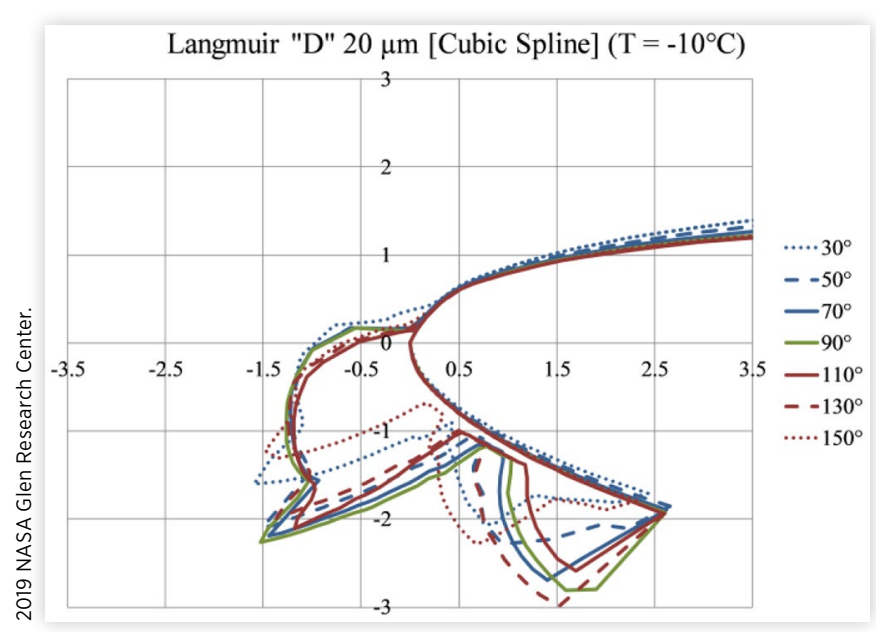

to the three Appendix O distributions seen in Fig. 9 that are characterized by a low collection efficiency.

Examining both the temperature sweeps in Fig. 11, as well as the sweeps of drop diameter distribution in Fig. 12, one can infer that the Langmuir " $D$ " $20 \mu \mathrm{m}$ distribution simulated at the $-10^{\circ} \mathrm{C}$ thermal condition generates the largest ice mass for the conditions simulated. The seven circumferential cuts of this condition are plotted in Fig. 13. The ice shapes are similar in size and shape for the seven circumferential cuts due to the relatively consistent collection efficiency around the inlet for this condition. The collection efficiency as a function of unwrapped distance can be seen in Fig. 14.

Although there is circumferential variation in the collection efficiency, the largest variation in the geometric features of the ice shapes for this distribution can primarily be attributed to the circumferential variation in the stagnation point.

FIGURE 14 Collection Efficiencies of Various Cut Planes for the Langmuir "D" $20 \mu \mathrm{m}$ Distribution.

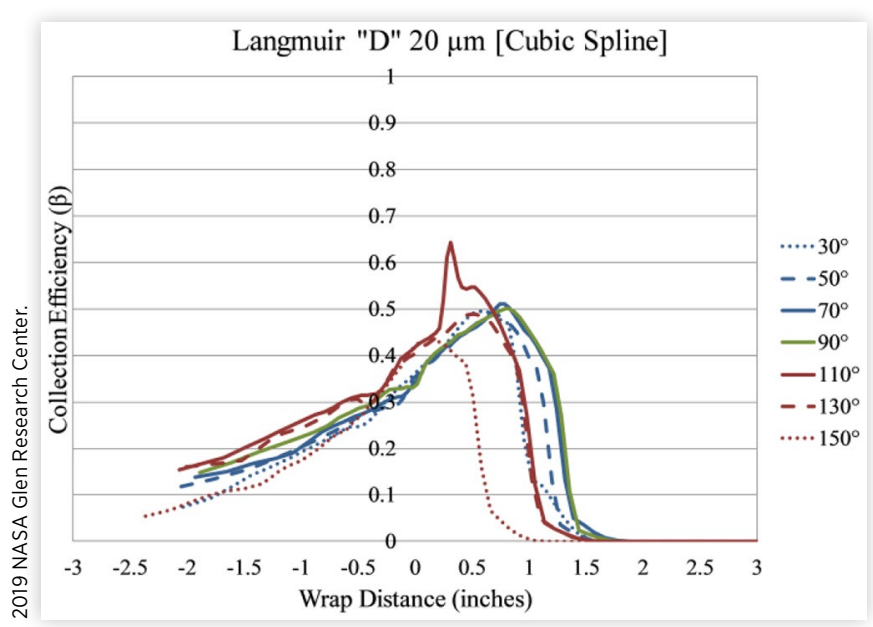


Computationally the location of the stagnation point in the two dimensional cut plane is the inflection point where runback changes from flowing aft along the upper surface or aft along the lower surface. For the vertical $\left(90^{\circ}\right)$ cut plane, the $4^{\circ}$ angle of attack of the aircraft causes the stagnation point to move aft on the lower surface of the engine inlet. However, it is the sideslip, not the pitch, which would cause the stagnation point to move for the horizontal cut planes. Since there is no sideslip, the stagnation point moves back towards the leading edge of the engine inlet as one moves circumferentially closer to the horizontal. This migration of the stagnation point greatly influences ice shape geometry of the two dimensional horns produced.

Although the Langmuir " $\mathrm{D}$ " $20 \mu \mathrm{m}$ distribution is characterized by relatively similar collection efficiencies across the circumferential range tested, this is not always the case. The drop sizes where the shadowzone are present on the engine inlet, i.e. drop diameters near the $47 \mu \mathrm{m}$ direct impingement cutoff, have an asymmetrical collection efficiency. This is evidenced by the striping of the collection efficiency in the Langmuir " $D$ " $40 \mu \mathrm{m}$ distribution (see Fig. 9). The striping trend is from a lower outboard to upper inboard due to the curvature of the nose geometry with the shadowzone being present below the striping. The effect of which is a lowering of collection efficiency at circumferential regions near the horizontal, favoring the inboard region. This can be seen in Fig. 15 where the $30^{\circ}$ and $50^{\circ}$ (inboard) as well as the $150^{\circ}$ (outboard) circumferential slices are marked by a significantly lower maximum collection efficiency.

Another effect of the shadowzones is that the concentration region that boarders the shadowzone region causes a spike in collection efficiency. This can be seen in the $110^{\circ}$ circumferential cut plane in Figs. $14 \& \underline{15}$. The spike for the Langmuir "D" $20 \mu \mathrm{m}$ condition is less significant than the spike for the Langmuir " $D$ " $40 \mu \mathrm{m}$ condition. As such the effect of the increased collection efficiency at this circumferential angle

FIGURE 15 Collection Efficiencies of Various Cut Planes for the Langmuir "D" $40 \mu \mathrm{m}$ Distribution.

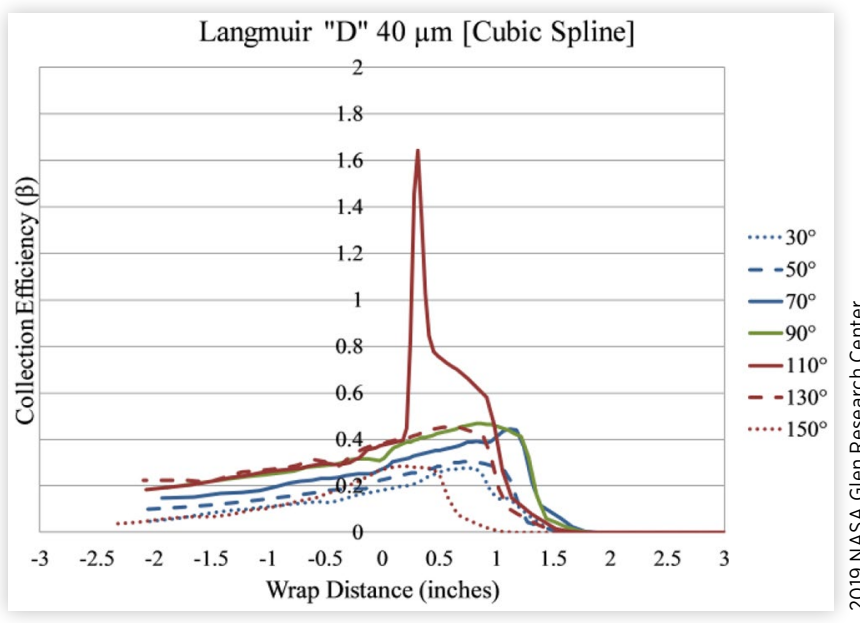

FIGURE 16 Simulated Ice Shapes of Various Cut Planes for the $-10^{\circ} \mathrm{C}$ Thermal Operating Condition and Langmuir " $\mathrm{D}$ " $40 \mu \mathrm{m}$ Distribution. Units of the Unlabeled Axes are Inches.

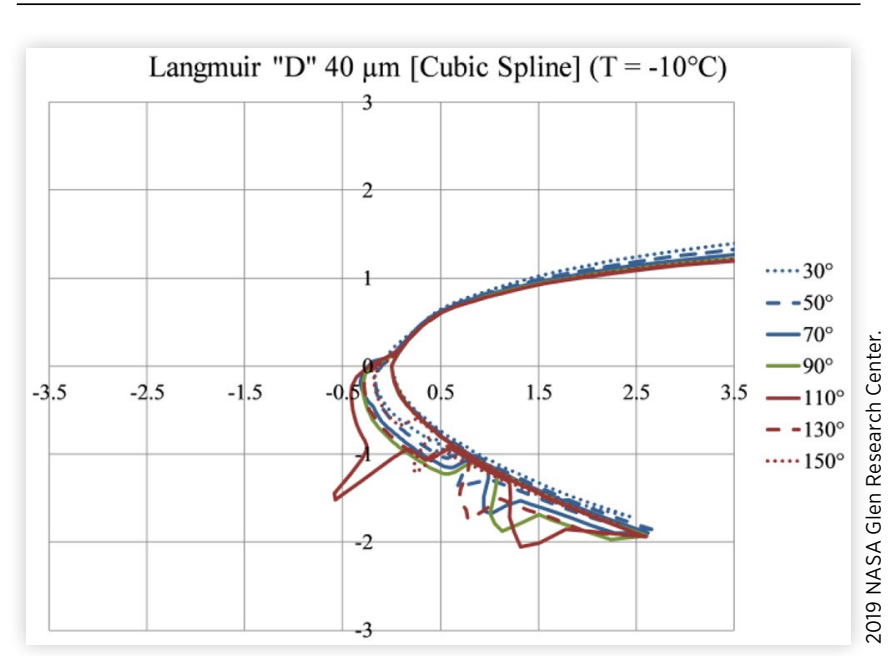

on the ice shape is not visually apparent for the Langmuir " $D$ " $20 \mu \mathrm{m}$ condition, i.e. the upper horn is slightly rounder on the upper side (see Fig. 13). However, the spike in collection efficiency for the Langmuir " $\mathrm{D}$ " $40 \mu \mathrm{m}$ distribution is so significant that there is a very apparent spike in the ice shape simulated (see Fig. 16). Note that this spike in collection efficiency will become smoother as the amount of discrete drop diameter bins goes to infinity.

\section{Summary}

Simulated ice shapes were generated for a variety of thermal conditions, cloud distributions and their associated liquid water contents. Liquid water content decreases as a function of temperature for each distribution. However, a majority of the liquid water remained unfrozen for the $0^{\circ} \mathrm{C}$ condition due to the high thermal content. As such, the largest ice shapes generated for each distribution occurred at the $-10^{\circ} \mathrm{C}$ condition due to the ability to freeze the impinged liquid water and the high liquid water content.

The distributions that generated the largest ice shapes were the Langmuir "D" $20 \mu \mathrm{m}$ and Freezing Drizzle MVD Less than $40 \mu \mathrm{m}$ distributions. These distributions were characterized by significant collection efficiency as well as high liquid water content. While the Langmuir “ $D$ ” $40 \mu \mathrm{m}$ distribution had considerable collection efficiency, its liquid water content for the Continuous Maximum cloud condition was significantly lower than all the other distributions.

The location of the stagnation point had significant impact on the two dimensional horn ice shapes generated. Due to the $4^{\circ}$ angle of attack of the aircraft, the stagnation point moved aft on the lower surface of the engine inlet for the vertical cut plane. The location of the stagnation point on 
the horizontal cut plane is effected by the sideslip, not the angle of attack, and therefore as one moves circumferentially towards the horizontal, the stagnation point moved towards the leading edge of the engine inlet.

Shadowzones of the larger drop diameters were present on the engine inlet. Due to the curvature of the nose of the aircraft, these shadowzones caused significant asymmetric collection efficiencies on the engine inlet for the Langmuir "D" $40 \mu \mathrm{m}$ distribution due to the considerable water content in this distribution at drop diameters that generated shadowzones on the engine inlet.

The concentration region directly neighboring the shadowzone region generated a striping of collection efficiencies on the engine inlet. Since the Langmuir " $D$ " $40 \mu \mathrm{m}$ condition contained considerable water content at drop diameters that generated shadowzones a significant spike was seen in the collection efficiency on one of the circumferential cuts. This spike was so significant that the simulated ice shape generated a considerable spike due to this high collection efficiency. This spike would likely be much smoother and less drastic as the amount of discretized bins goes towards infinity.

No validation data exists for the splashing, re-entrainment, and subsequent re-impingement of the re-entrained splashed liquid water. As such these results were run absent of the splashing model in LEWICE3D, i.e. they depict direct impingement only. The inclusion of splashing effects would likely increase the amount of water that impinges the engine inlet, especially for the Appendix $\mathrm{O}$ conditions that contain considerable water content at large drop diameters. However, with zero experimental data available to benchmark the splashing model, the efficacy of the model is completely unknown, and hence neglected.

\section{References}

1. Del Rosario, R. et al., "Subsonic Fixed Wing Project Overview of Technical Challenges for Energy Efficient, Environmentally Compatible Subsonic Transport Aircraft," in 50th AIAA Aerospace Science Meeting, Nashville, TN, 2012; Guo, Q. and Liu, B., "Simulation and Physical Measurement of Seamless Passenger Airbag Door Deployment," SAE Technical Paper 2012-01-0082, 2012, doi:10.4271/2012-01-0082.

2. Bidwell, C.S., "User's Manual for the NASA Glenn ThreeDimensional Grid Based Ice Accretion Code (LEWI3DGR Ver. 1.7)," 2005.

3. Wright, W.B., "User Manual for the NASA Glenn Ice Accretion Code LEWICE. Version 2.2.2," 2002.

4. Title 14 Code of Federal Regulations, Aeronautics and Space, Part 25 Airworthiness Standards: Transport Category Airplanes, Section C25.1: Appendix C; published by the Office of the Federal Register, National Archives and Records Administration, Washington, DC., Amdt. 25-140, effective 5-Jan-2015.
5. Title 14 Code of Federal Regulations, Aeronautics and Space, Part 25 Airworthiness Standards: Transport Category Airplanes, Section O25.1: Appendix O; published by the Office of the Federal Register, National Archives and Records Administration, Washington, DC., Amdt. 25-140, effective 5-Jan-2015.

6. Bidwell, C.S., "Icing Analysis of a Swept NACA 0012 Wing Using LEWICE3D Version 3.48," in AIAA Aviation Forum, Atlanta, GA, 2014.

7. Bidwell, C.S. "Super Cooled Large Droplet Analysis of Several Geometries Using LEWICE3D Version 3," in AIAA Atmospheric and Space Environments Conference, Toronto, ON, 2010.

8. Papadakis, M. et al., "Comparison of Experimental and Computational Ice Shapes for an Engine Inlet," in AIAA Atmospheric and Space Environments Conference, Toronto, ON, 2010.

9. Bidwell, C.S., "Icing Calculations for a 3D, High-Lift Wing Configuration," in 43rd AIAA Aerospace Sciences Meeting and Exhibit, Reno, NV, 2005.

10. Bidwell, C., "Icing Analysis of the NASA S3 Icing Research Aircraft Using LEWICE3D Version 2," SAE Technical Paper 2007-01-3324, 2007, doi:10.4271/2007-01-3324.

11. Greitzer, E.M. et al, "N+3 Aircraft Concept Designs and Trade Studies, Final Report,” NASA CR-2010-216794/Vol.1, 2010.

12. Nichols, R.H. and Buning, P.G., "User's Manual for OVERFLOW 2.2," University of Alabama and NASA Langley Research Center, 2015.

13. Menter, F., "Zonal Two Equation $\kappa-\omega$ Turbulence Models for Aerodynamic Flows," in 24th AIAA Fluid Dynamics Conference, Orlando, FL, 1993.

14. Messinger, B.L., "Equilibrium Temperature of an Unheated Icing Surface as a Function of Air Speed," Journal of the aeronautical sciences 20(1):29-42, 1953.

15. Langmuir, I. and Blodgett, K., "Mathematical Investigation of Water Droplet Trajectories," AAFTR 5418, 1946.

16. Heinrich, A. et al., "Aircraft Icing Handbook, Volume I, DOT/FAA/CT-88/8-1, 1991.

17. Papadakis, M. et al., "Large and Small Droplet Impingement Data on Airfoils and Two Simulated Ice Shapes," NASA/ TM-2007-213959, 2007.

18. Porter, C.E. and Potapczuk, M.G., "Computational Icing Risk Analysis of the D8 'Double Bubble' Aircraft,” in 2018 Atmospheric and Space Environments Conference, AIAA Paper 2018-2859, NASA/TM-2018-220006, 2018.

\section{Acknowledgments}

The authors would like to thank NASA's AATT project for its financial support of this research effort, as well as H. Doğuş Akaydin and Shishir Pandya of NASA Ames Research Center for their efforts in the grid generation and CFD simulation of the representative hold condition for the D8 "Double Bubble" geometry. 


\section{Definitions/Abbreviations}

AoA - Angle of Attack

B (beta) - Local Collection Efficiency

CFD - Computational Fluid Dynamics

$\mathbf{d}_{\mathbf{i}}$ - Discrete Drop Diameter

FAA - Federal Aviation Administration

LWC - Liquid Water Content

$\mathbf{M}_{\infty}$ - Freestream Mach Number

MIT - Massachusetts Institute of Technology
MVD - Median Volume Diameter

NASA - National Aeronautics and Space Administration

$\mathbf{R e}_{\infty}$ - Freestream Reynolds Number

SLD - Supercooled Large Drops

SST - Shear Stress Transport (Turbulence Model)

TWC - Total Water Content

$\boldsymbol{\mu m}$ - micron/micrometer

$\mathbf{V}_{\infty}$ - Freestream Velocity

2019 NASA Glen Research Center. This is the work of a Government and is not subject to copyright protection. Foreign copyrights may apply. The Government under which this work was written assumes no liability or responsibility for the contents of this work or the use of this work, nor is it endorsing any manufacturers, products, or services cited herein and any trade name that may appear in the work has been included only because it has been deemed essential to the contents of the work.

Positions and opinions advanced in this work are those of the author(s) and not necessarily those of SAE International. Responsibility for the content of the work lies solely with the author(s).

ISSN 0148-7191 